\title{
Pour une bibliothèque virtuelle universelle
}

\author{
Christian Vandendorpe
}

Université d’Ottawa

Article publié dans Le Débat, no 117, novembre 2001, p. 31-42

Même si la frénésie des années 90 s'est quelque peu calmée, le Web reste un enjeu économique majeur qui suscite bien des convoitises et bien des incertitudes. Parmi les visions antagonistes qui s'opposent sur les orientations à donner à ce nouvel espace, l'une est d'en faire une zone sévèrement contrôlée, qui ne serait que la doublure virtuelle du monde physique et où quelques grands fournisseurs de contenus pourraient maximiser les ventes de "contenus ", à savoir de produits culturels. Sans être opposé à une exploitation commerciale qui semble inévitable voire souhaitable, il me paraît cependant essentiel de préserver le caractère public de cet espace et d'adapter nos lois en matière de propriété intellectuelle afin qu'il puisse pleinement remplir son rôle de bibliothèque universelle et contribuer à l'avancement du " bien commun mondial ", selon l'expression de Philippe Quéau [1].

Au cours des années soixante, l'Américain Ted Nelson avait envisagé un vaste hypertexte, appelé Xanadu, grâce auquel les éditeurs participants pourraient rendre accessibles sous format numérique les ouvrages de leurs collections, moyennant un système de micropaiements [2]]. Il donnait ainsi une forme électronique et marchande à un rêve caressé dès la fin de la deuxième Guerre mondiale par Vannevar Bush, qui visait à reproduire sur microfiches photographiques tout le savoir de l'humanité, afin de le rendre facilement accessible aux chercheurs []] . Le projet de Nelson, qui ressemblait d'abord à une utopie techno-commerciale, a fait place dans la dernière décennie à la réalité du Web, lequel pourrait en apparaître comme la réalisation idéale, s'il n'offrait pas la particularité d'être gratuit -- développement dont peu de personnes entrevoyaient naguère la possibilité. Malgré cet échec relatif de son modèle commercial, le visionnaire américain, qu'un article de The Economist surnommait " The Babbage of the web ", n'a pas baissé les bras et continue à militer pour un système dont le principal mérite est, de l'avis du même magazine, " qu'il facilite le suivi de l'utilisation et la gestion des droits, et qu'il peut former la base d'un système de royautés" []].

Quoi que l'on puisse penser de ce modèle, qui a été comparé à un McDonald's de 
l'information [్], il est parfaitement compatible avec la tendance dominante de la nouvelle économie exposée par J eremy Rifkin []]. Au lieu d'accumuler et de vendre des objets matériels, les compagnies cherchent aujourd'hui à vendre des droits d'accès et de participation. Ce nouveau souffle du capitalisme a bénéficié d'un élan extraordinaire avec la rapide expansion du réseau Internet. Depuis quelques années, les géants du logiciel comme Microsoft étudient très sérieusement la possibilité de ne plus vous vendre un traitement de texte, mais simplement de vous le louer sur le Net. Dès lors que la plupart des ordinateurs seront connectés à l'Internet, une telle solution pourrait bien devenir inévitable, avec ou sans le consentement des usagers. Tout étant question d'habitude et donc d'éducation, la clientèle des jeunes fait l'objet d'une sollicitude particulière de la part des producteurs et une part croissante des jeux sur ordinateur ne peuvent déjà plus se jouer que sur Internet moyennant une redevance mensuelle. D’après Rifkin, ce qui est valable pour le logiciel l'est aussi pour la totalité des données susceptibles d'être transmises sur les réseaux. Cela explique sans doute la frénésie de fusions qui ont abouti à la formation des conglomérats que sont AOL-Time Warner, Bertelsmann et Vivendi Universal. Les contraintes imposées par la mise en place des infrastructures et la gestion d'immenses parcs d'abonnés sont telles que seuls de très grands groupes seront en mesure d'imposer leur standard dans ce nouvel espace et de harnacher l'énorme potentiel économique d'un réseau couvrant l'ensemble de la planète. Dans leur quête de " contenus " à offrir, on peut craindre que ces géants ne fassent bientôt passer l'ensemble de la sphère culturelle sous la loi du marché avec la complicité passive -- et parfois même active -- des gouvernements, mettant ainsi un terme brutal au modèle séculaire de la bibliothèque.

L'ordinateur, en offrant à travers une fenêtre unique livres, chansons et vidéos, tend à homogénéiser le statut des différents arts, puisque tout y est finalement soumis aux mêmes manipulations. Les effets de cette convergence sur le statut de l'activité de lecture sont déjà visibles. Celle-ci, telle que nous la connaissons dans le monde physique de l'imprimé, est par excellence une activité privée, aux rythmes mouvants et incertains, tantôt rapide, tantôt lente et méditative. $\mathrm{Au}$ contraire, lorsqu'elle s'exerce sur du texte numérique, elle est presque obligatoirement ciblée et emportée par le cliquetis de la souris sur les liens hypertexte, la structure fragmentée du texte et la position rigide de lecture imposée par le médium invitant à sauter rapidement d'un point à un autre. Ces contraintes conviennent parfaitement à une lecture " ergative ", orientée en vue d'une action à effectuer ou de réponses à trouver; elles sont totalement inadaptées à la lecture de fond, qui consiste à accueillir en soi une pensée neuve et complexe ou à plonger dans un univers romanesque. Si l'on ajoute à cela que, pour séduire le lecteur, le texte se fait chatoyant en recourant à des couleurs, des icônes, des images, la lecture tend à être déportée vers l'ordre du spectacle. Ceest 
d'ailleurs à un spectacle que nous convient nombre de sites, dont certains présentent à leur entrée des séquences d'animation fort élaborées tandis que d'autres vont jusqu'à faire défiler les pages en mode automatique. Or le spectacle moderne relève par excellence de la consommation, ainsi que l'avait pressenti Guy Debord: "Le spectacle est le moment où la marchandise est parvenue à l'occupation totale de la vie sociale" [7]. Après avoir été pour notre civilisation l'incarnation du " logos " et sa raison suprême, le texte est devenu un enjeu idéologique majeur de la société néolibérale, qui est d’assurer le recouvrement complet du culturel par le commercial.

Cet objectif s'est cristallisé sur le resserrement des notions de droits de reproduction, de copie privée et même de droit de lecture, à la faveur de la rupture introduite par le nouvel environnement virtuel. Dans le monde physique, les droits de propriété des éditeurs sont protégés en partie par les obstacles matériels que pose la reproduction, car il est onéreux en temps et en équipement physique de dupliquer une cassette ou de photocopier un livre. En outre, l'original perd beaucoup de sa qualité au cours du processus. Cest cette inertie et cette entropie du monde physique qui ont permis au système en vigueur de fonctionner avec une marge de jeu généralement jugée satisfaisante depuis plus de deux siècles. La situation est tout autre dans le nouvel univers numérique, où n'existe aucune sorte de friction ni marge de jeu. À partir du moment où une production culturelle est numérisée, par exemple une pièce musicale, elle acquiert de par son immatérialité une mobilité extraordinaire, qui permet de la transporter d'un bout à l'autre de la planète en un instant et pour un coût pratiquement nul. Elle peut aussi, en principe, être copiée avec une extrême facilité. L'usager pourrait dès lors jouir de ces productions sans que les auteurs et éditeurs soient en mesure de percevoir leurs droits et il serait d'autant plus tenté de le faire que la chose est facile et presque " normale".

La gratuité semble en effet être au coeur de la nouvelle culture. Ayant déjà à acquitter le coût d'une connexion par modem ou par câble, l'usager répugne à payer un supplément pour un service automatisé qui ne semble entraîner de la part du fournisseur aucune dépense, à la différence d'une transaction dans le monde matériel, où sont impliqués des objets ou des espaces physiques. Cette situation pourrait certes se modifier avec l'avènement d'un réseau Internet de nouvelle génération, qui serait capable de différencier les fichiers échangés selon leur contenu, et où l'usager se verrait offrir des débits variables en fonction du prix payé [] ]. Celui-ci paierait alors pour le service combiné d'une liaison rapide et d'un contenu exigeant en bande passante, tel un vidéo, ainsi qu'il le fait déjà quand il se connecte par l'intermédiaire d'un téléphone mobile, d'un service de satellite ou de câblodistribution. Mais, quand seul est en jeu un contenu immatériel, c'est pour le moment le libre accès qui est la norme. Cette situation 
est due en partie à des circonstances historiques, le nouveau médium étant né au sein de la communauté scientifique et s'étant développé dans des pays où les communications téléphoniques locales ne sont pas tarifées à la minute. Cette culture de la gratuité s'est consolidée en raison de la nature profondément égalitaire de l'Internet, qui permet à chacun de créer son propre site Web et, en devenant à son tour fournisseur de contenus, de s'emparer d'une prérogative jusque là réservée à des sociétés disposant de capitaux imposants. Chaque jour, des milliers d'internautes mettent bénévolement leur savoir au service d'autrui, que ce soit en plaçant sur le Web des documents de toute sorte, du livre de recettes au journal personnel, ou en rédigeant des articles d'opinions sur tous les produits et questions imaginables pour des sites de discussion [9]]. De même, les universités et les agences gouvernementales déposent une masse considérable de données, parfois d'un très grand intérêt. Le savoir ainsi partagé a atteint un tel volume que l'Encyclopaedia Bitannica a jugé utile d'ajouter à ses propres articles des références à des sites Web [10]. Devant cette concurrence spontanée qui leur est faite pour capter l'attention des lecteurs et occuper le discours public, les journaux ont bien été forcés d'entrer en lice. Après avoir cru pouvoir exiger un abonnement de la part des lecteurs, ils ont dû y renoncer les uns après les autres, sans pour autant abandonner l'espace virtuel. Une présence forte sur le Web est en effet aujourd'hui aussi indispensable à la crédibilité et au dynamisme d'une entreprise de presse que pouvait l'être naguère un prestigieux édifice pour abriter ses locaux.

En réponse à cette idéologie du partage, les intérêts éditoriaux ont durci leurs positions, d'abord en faisant adopter aux États-Unis le Digital Millenium Copyright Act (1998), lequel a été suivi par des législations similaires en Europe [11]. Puis est venue la grande offensive contre Napster, menée par les éditeurs de musique. Dans la foulée, il ne se passe pas de mois sans que soient annoncés des systèmes sophistiqués au moyen desquels le producteur pourra restreindre l'accès aux données et exiger un droit de péage des Internautes. C'est ainsi que Microsoft tente de se gagner les fournisseurs de contenus en imposant avec Windows Media Player 8 un nouveau standard de compression de fichiers musicaux, le WMA, qui remplacerait le populaire MP3. L'avantage de ce système pour l'industrie est qu'il rend possible le cryptage permanent d'une pièce musicale à l'exception du moment précis où elle passe par la carte de son. Ce procédé permettra d'en restreindre l'exécution sur la seule machine pour laquelle la pièce aura été achetée, et seulement pour la durée prévue par la licence. Des mécanismes similaires rendront aussi l'échange ou la copie de livres impossible, limitant l'usage que pourrait en faire le client qui se les serait procurés. C'est le cas par exemple des ouvrages électroniques créés pour le Glassbook Reader, qui ne peuvent être lus que sur la machine qui a servi à leur achat. Même le légitime propriétaire n'en a pas la pleine jouissance, car il ne 
peut pas les transférer d'un de ses ordinateurs à un autre, ni les récupérer lejour où son disque dur cesserait de fonctionner. Le consommateur culturel sera donc devenu éternel locataire. Des opérations de copier/coller, qui sont un des principaux atouts de l'environnement électronique, pourraient aussi être rendues impossibles, même à des fins parfaitement légales de copie privée et de " fair use ", ainsi que l'a fait remarquer un juriste spécialisé dans ces questions [12]. En matière d'abonnements virtuels, on voit déjà apparaître des systèmes limitant le nombre de pages susceptible d'être imprimé d'un texte emprunté, comme le fait le réseau NetLibrary aux États-Unis. Même la très conservatrice revue The Economist s'est inquiétée du fait que les succès remportés auprès des législateurs par les lobbies de l'édition pourraient mettre en danger des droits existants, comme celui de faire des copies pour son usage personnel [13]. Plus grave encore, l'avantage juridique donné aux détenteurs de droits pour défendre leur propriété encourage la mise en place de mécanismes de surveillance omniprésents, qui menacent le droit de l'individu à sa vie privée. Divers programmes permettant déjà de suivre à la trace le chemin parcouru par un fichier, on n'est pas loin de pouvoir ficher les habitudes de consommation virtuelle de tout un chacun. En criminalisant les activités de copie personnelle, les nouvelles lois relatives à la protection intellectuelle pourraient ainsi favoriser l'avènement d'un monde orwellien, dans lequel les opérations fondamentales de la culture seraient soumises à la traçabilité permanente de tous les usagers des réseaux. L'auteur de 1984 devrait apprécier l'ironie d'une situation où la protection des droits de l'écrivain est invoquée pour brimer ceux du lecteur et de la société tout entière. Voilà déjà quelques années, extrapolant sur les procédés susceptibles d'être mis en place afin d'assurer le respect des droits d'auteur, Richard Stallman avait créé une fable intitulée " Le droit de lire " [14], dont le pessimisme ne le cède en rien à celle d'Orwell. Ces dangers ne semblent pas préoccuper sérieusement les pouvoirs publics, que séduisent surtout les perspectives de croissance économique.

Dans un ouvrage bien informé qu'il vient de consacrer à la propriété intellectuelle sur Internet, le juriste Bertrand Warusfel essaie d'offrir une vue balancée entre les tendances à la dérégulation et un renforcement de la propriété intellectuelle. Il reconnaît que les nouvelles mesures législatives demandées par l'industrie auraient des conséquences étendues et que " le contrôle technique et juridique des droits d'usage pourrait entraîner une mutation philosophique profonde du concept de propriété intellectuelle " [15]. On observe ainsi depuis quelques années une tendance à fusionner les concepts de brevet et de droit d'auteur, à la suite de glissements progressifs, favorisés notamment par l'industrie du logiciel et la nouvelle économie. La principale différence entre brevet et droit d'auteur réside dans les droits accordés à l'usager. Alors que le droit d'auteur ne s'opposait pas directement au droit d'usage, le brevet permet 
d'interdire l'emploi d'un procédé à quiconque n'en est pas le légitime propriétaire. On est loin ici de la réflexion sur le plagiat élaborée dans la sphère littéraire, où la préoccupation première est d'ordre esthétique et qualitatif, et vise à faire reconnaître la paternité d'un écrivain sur sa création, en affirmant son antériorité et son originalité; les poursuites en matière de brevet reposent sur un critère économique et visent à protéger un investissement.

Cette contamination du concept de droit d'auteur par celui de brevet a commencé à pénétrer les conceptions courantes. C'est ainsi qu'il est fréquent de voir assimiler à du pillage le simple fait de lire ou d'écouter gratuitement une œuvre. Dans une réflexion par ailleurs très juste sur les nouveaux comportements de consommation culturelle, un journaliste du Monde écrivait ainsi récemment : " l'internaute ne se contente pas de piller, il est aussi devenu un véritable producteur de gratuité” [16]. L'infamie attachée au vol est donc lancée sur toute personne qui jouirait librement des œuvres culturelles. Voilà qui n'est pas sans rappeler le cas du “ Miserere ” d'Allegri, dont le pape Urbain VIII et ses successeurs avaient interdit la copie et réservé l'exécution à la seule enceinte de la chapelle Sixtine. Il aura fallu la mémoire prodigieuse de Mozart enfant, admis à l'écouter, pour que cette pièce puisse enfin passer dans le domaine public. Il est probable que, aujourd'hui, avec les nouvelles lois protégeant la propriété intellectuelle, un tel exploit vaudrait au jeune prodige d'être arrêté pour " copie illicite " et " diffusion sans autorisation d'une œuvre protégée ". Là encore, les papes invoqueraient sans doute leur droit de " protéger un investissement ", vu que le compositeur avait été engagé sous contrat. Un autre exemple de cette dangereuse dérive mercantile a été offert par le récent débat sur le prêt payant en bibliothèque. Interrogé à ce sujet, Daniel Pennac avait relevé l'aberration qu'impliquait de la part d'un écrivain l'invocation du " droit à ne pas prêter ses livres " [17]. A tout prendre, ne vaudrait-il pas mieux aller dans le sens de J ean-Marie Laclavetine, selon qui “ le droit essentiel de l'auteur, c'est celui d'être lu " [18] ?

Ces débats confirment la pertinence de l'analyse de Rifkin sur le conflit opposant la sphère culturelle et la sphère commerciale pour contrôler l'accès aux contenus [19]. Les intellectuels ne devraient pas accepter comme une évidence ou une fatalité le mouvement d'idéologie néolibérale qui tend à faire passer dans la sphère marchande la totalité des activités humaines [20]. Une œuvre littéraire n'est pas une marchandise comme une autre. Loin d'être une création ex nihilo, elle est fabriquée à même les mythes d'une société, ses acteurs, ses angoisses, les événements qui la secouent. En cela, elle ne saurait être la seule et exclusive propriété de l'écrivain, mais appartient aussi, au moins partiellement, au public en vue duquel elle a d'abord été créée et dont procède sa valeur d'échange. Si les lois reconnaissent le droit d'un individu à son image, il ne serait pas abusif de 
reconnaître aussi certains droits à une société sur les œuvres qu'elle a inspirées. De même, dans un domaine voisin, le mouvement de restitution engagé par les musées nationaux envers les anciennes puissances coloniales témoigne de ce qui peut être vu comme une relation originelle de complémentarité entre l'artiste et son public. Il faut reconnaître, enfin, que certains droits individuels ne peuvent s'exercer que dans un cadre social : le créateur ne peut ouvrer et jouir de ses productions que dans la mesure où la société lui confère un " espace culturel " approprié, à l'intérieur duquel il pourra retirer du public les récompenses que lui mérite son travail. Cette interdépendance entre le créateur et la société est suffisamment établie pour ne pas faire du droit d'auteur un absolu et justifier que le législateur limite les modes d'appropriation des ouvres culturelles en ayant en vue l'épanouissement des arts et l'intérêt du public.

L'œuvre littéraire, qui a pour fonction ultime de donner du sens à l'existence, est un enjeu social, ce qui suppose qu'on puisse en parler, en citer des extraits, la parodier surtout et la pasticher, voire la plagier, ainsi qu'y invitaient Anatole France et à sa suite J ean-Luc Hennig [21]. À partir du moment où la lecture ne peut plus se partager, c'est la condition fondamentale et le rôle premier de la culture qui sont en cause. Accepter de placer la lecture sous la coupe de l'économie marchande accélérerait fatalement le laminage des productions culturelles en faveur du divertissement et de la production de masse, qui seule deviendrait accessible à tous grâce à la sponsorisation des publicistes. Le législateur ne devrait donc pas céder à des intérêts privés un domaine aussi important que celui de la culture ni tolérer que soit créée une pénurie artificielle de biens culturels en transposant dans le monde virtuel des barrières économiques que seules des contraintes matérielles pouvaient justifier dans le monde physique.

Des décisions juridiques récentes auraient établi, selon les termes de Bertrand Warusfel, que " l'Internet ne présente pas de particularité de principe " par rapport au monde physique " puisque tout acte d'exploitation non autorisé d'une œuvre protégée sur l'Internet va être considéré comme une contrefaçon " [22]. Une telle position ignore complètement la spécificité de la lecture sur écran, qui n'est en rien assimilable à la lecture du livre, ce dont tout un chacun est en mesure de témoigner en raison d'expériences mille fois corroborées [23]. Il existe un gouffre entre le monde virtuel et le monde physique, ainsi que l'a appris à ses dépens l'auteur américain Stephen King, qui avait cru en juin 2000 pouvoir court-circuiter son éditeur en rendant disponible sur Internet, par tranches mensuelles, un nouveau roman intitulé The plant. L'affaire tourna court et le roman fut abandonné en décembre de la même année, après seulement six chapitres, l'écrivain ayant vu fondre son lectorat de façon continue [24]. Si cet auteur de bestsellers n’a pas réussi à garder ses lecteurs sur écran avec des 
histoires à suspense aussi bien ficelées que les siennes, on peut se demander en quoi la mise en ligne des textes de Sartre, Queneau ou Lautréamont menacerait les ventes de leurs œeuvres dans le monde physique. L'insistance de Warusfel sur le fait qu'il n'y aurait pas de différence entre l'espace du Web et le monde du livre est d'autant moins compréhensible que le même juriste reconnaît ailleurs que la photocopie à usage privé constitue une exception normale au régime du droit d'auteur, car cette “ tolérance juridique [...] n'avait que peu de conséquences pratiques et économiques dans le monde analogique, la photocopie d'un livre ne remplaçant pas vraiment l'achat du volume lui-même " [25]. Si la photocopie ne saurait remplacer le livre, on voit mal en quoi une copie sur écran ou sur imprimante pourrait être jugée équivalente à ce dernier.

Nombre de juristes sont cependant sensibles aux différences entre le monde physique et le cyberespace [드] et des centaines de professeurs de droit ont signé aux États-Unis une pétition mettant en garde contre l'adoption du Digital Millenium Copyright Act [27]. Dans la foulée, on a aussi remis en cause l'argument souvent avancé selon lequel, sans un contrôle extrêmement strict des droits d'auteur, les créateurs perdraient toute motivation économique et la création se tarirait. Cet argument ne tient pas compte de la réalité psychologique et sociale qui est à la base des productions artistiques. Il n'est pour s'en convaincre que de considérer la somme énorme de travail purement gratuit investi dans la production de sites personnels aussi attrayants et originaux que possible, et explorant de nombreux registres de la création artistique. L'observation ancienne de Cicéron selon laquelle " l'honneur est l'aliment des arts " [28] est toujours vraie. En dépit de la sophistication de notre économie, l'attention d'autrui constitue le levier psychologique ultime. L'espace du Web, avec les divers outils permettant à un auteur d'évaluer sa notoriété (compteurs de visites, renvois à ses pages, modes d'interactivité, distinctions diverses), a fait de cette récompense psychologique un stimulant à la création plus puissant et plus efficace que tout ce qui existait auparavant. L'hypothèse selon laquelle un relâchement des droits d'auteur dans cet espace entraînerait un effondrement de la création ne résiste donc pas à l'analyse. Comme le notent les juristes D.R. J ohnson et D. Post :

La justification de base pour la protection du droit d'auteur réside dans l'hypothèse qu'en accordant aux auteurs le droit exclusif de contrôler la reproduction et la distribution de leurs œeuvres on augmentera la production de ces œuvres grâce à la récompense monétaire qui leur est ainsi offerte en contrepartie de l'effort exigé par le travail de création. Mais même dans le " monde réel ", une large part des œuvres de création est complètement indépendante d'un tel type de récompense, parce que la première récompense de 
l'auteur dépend davantage de son acceptation par une communauté et de la réputation acquise par une large dissémination de son œuvre que du fait de licencier et de vendre des copies de son œuvre. Et cette observation est sans doute encore plus vraie dans le cyberespace [29].

Est-il nécessaire en outre de rappeler que des auteurs très créatifs ne retirent guère de droits par manque d'une commercialisation adéquate de leurs œuvres tandis que d'autres, bénéficiant d'une position dominante dans la distribution, amassent des fortunes avec des créations insignifiantes et produites en séries ? Selon toute probabilité, le mouvement de globalisation va renforcer à l'extrême cette inégalité et laisse craindre que, loin de favoriser la diversité culturelle, les dispositions actuelles appliquées à la Toile serviront à la constitution d'immenses conglomérats de distribution imposant des produits standardisés.

Au cours des dernières années, une réflexion intéressante s'est développée sur ces questions autour de la revue Wired et de la Electronic Frontier Foundation, notamment avec les écrits de John Perry Barlow [30]. Pour ce dernier, nous entrons dans une nouvelle forme d'économie, basée sur l'attention et la notoriété. Dans celle-ci un auteur connu ou un chanteur célèbre pourront continuer à vivre très bien, non pas en vendant des objets virtuels, mais en monnayant leur participation à des événements dans le monde physique. Loin d'être marginales, ces positions sont de plus en plus largement partagées. Il serait souhaitable que la sphère politique soit plus attentive à ce nouveau climat de pensée, au lieu de légiférer à la hâte sous la pression de groupes d'intérêt. Même si, de par sa nature, le domaine du droit est extrêmement conservateur, et donc porté à renforcer les dispositifs protégeant les détenteurs de droits plutôt qu'à les restreindre, il ne devrait pas se faire l'allié objectif des seuls intérêts privés et étouffer sous un appareil législatif et juridique d'un autre âge la révolution de l'information.

Car c'est bien d'une révolution qu'il s'agit ici, et qui est susceptible de modifier radicalement la façon dont on travaille et dont on participe à la vie culturelle. Pour la première fois dans l'histoire de l'humanité, la totalité des ouvres de l'esprit pourrait en principe être accessible de partout, sans délai d'attente et pour un coût global de connexion assez faible. Mieux encore, des outils de recherche de plus en plus performants, tels Google et Copernic, permettent de trouver en quelques instants un renseignement précis ou de recueillir une masse de données sur n'importe quelle question. En comparaison, la bibliothèque des années 60 était d'une lenteur et d'une insuffisance intolérables ! Après avoir entrevu un tel paradis pour la recherche et constaté ses effets sur l'accélération des connaissances, les usagers du domaine scientifique ne peuvent déjà plus envisager un retour en arrière, mais exigent au contraire une accélération du 
mouvement, et que soit mise en ligne la totalité du savoir spécialisé. C’est dans cet esprit, et afin de contrer le mouvement de privatisation du savoir, que le très influent MIT a annoncé en avril dernier sa décision de rendre accessible gratuitement sur le Web tout le contenu de ses cours d'ici dix ans [31]. Plus significative encore est l'initiative lancée aux États-Unis par des chercheurs en biologie en vue de constituer une " bibliothèque publique des sciences ". Dans une lettre ouverte qui avait déjà recueilli quelque 22000 signatures en mai 2001, ce groupe réclamait que les archives de la recherche scientifique soient placées en accès libre dans des bibliothèques publiques internationales contenant le texte complet de tous les articles publiés, dans un format qui soit ouvert aux moteurs de recherche et parfaitement échangeable [32]. Plusieurs éditeurs de revues scientifiques ayant déjà accepté de mettre en ligne leurs articles six mois après leur publication, le groupe Reed Elsevier, qui contrôle plus de 1700 titres, se disait alors prêt à étudier un compromis.

Cet élan en faveur d'une bibliothèque virtuelle universelle doit-il se limiter aux sciences ? Dans l'état actuel des mentalités, on peut craindre que les éditeurs de littérature et de sciences humaines soient beaucoup moins prêts que leurs confrères des sciences biologiques à lâcher du lest. Certains diront sans doute que le fétichisme du droit d'auteur y est trop solidement établi et le besoin de la libre consultation des textes moins pressant. En acceptant un tel raisonnement, on risque de consacrer la marginalisation de tout ce champ et d'en accélérer le déclin dans une société imprégnée de technicité. Compte tenu de l'importance croissante que prend le Web dans les démarches d'accès à l'information, on ne devrait pas accepter qu'un pan entier de la culture reste confiné au monde statique de l'imprimé ou qu'il dorme dans des bases privées sans pouvoir jouer pleinement son rôle dans les nouvelles créations et les recherches en cours.

La principale difficulté réside dans le fait que, à la différence des auteurs d'articles de revues scientifiques qui ne perçoivent normalement pas de droits d'auteur, les écrivains dépendent de ces revenus pour vivre, en tout ou en partie. C'est pour cette raison que la législation française a étendu la durée de protection de façon continue. Limitée à dix ans lors de l'établissement du droit d'auteur en 1791, cette période a été étendue à 20 ans en 1810, à 30 en 1854, et à 50 en 1866. En 1957, elle était de 50 ans après le décès du créateur. A la suite d'une directive européenne, cette durée a été étendue à 70 ans; elle vient d'être portée à 95 ans aux États-Unis avec l'adoption du Digital Millenium Copyright Act. En même temps que s'allongeait la durée de protection, c'est le concept même de domaine public qui se réduisait chaque fois davantage, comme peau de chagrin, au point de n'avoir bientôt plus aucune signification.

Sans vouloir dénigrer le droit d'auteur, dont je bénéficie moi-même de façon modeste, il faut cependant rappeler que celui-ci ne profite pas d'abord à l'auteur, 
mais à la société éditrice, qui perçoit la plus grande part des produits de la vente des livres ou des disques. Ce partage inégal pouvait se justifier dans le monde des échanges physiques. Dans un régime numérique où les coûts de distribution et de copie sont pratiquement inexistants, on peut soupçonner que la mise en avant du droit d'auteur relève d'une stratégie concertée des lobbies de l'édition pour justifier des grilles tarifaires sans commune mesure avec les coûts réels et donner une façade de légitimité à une opération purement commerciale. Le capital symbolique associé à la notion de droit d'auteur, en faveur duquel vient même d'être créée une " J ournée internationale ", se révèle ainsi pour le monde des affaires un fort efficace cheval de Troie dans sa tentative de mainmise sur la culture. C'est également le droit d'auteur qui est mis de l'avant par les producteurs de DVD pour justifier le découpage de la planète en quelques zones aux protocoles incompatibles : cette pratique qui a attiré l'attention du bureau de la concurrence de la Commission européenne est enfin soupçonnée de n'être qu'un " écran de fumée " par le commissaire Mario Monti [33]. Dans le désir bien compréhensible de protéger leur position dominante, des compagnies comme Microsoft, qui ont accumulé des fortunes colossales grâce aux droits de propriété intellectuelle sur un système d'exploitation devenu standard, ne manquent pas une occasion de faire l'apologie de ce droit et même de dénoncer comme une infection " virale " et un " cancer " l'idéal de gratuité qui anime Linux et le courant du logiciel libre, dit "Open Source" [녀]. Quand on sait par ailleurs que cette même compagnie a tenté d'acheter par sa filiale Corbis les droits exclusifs de reproduction des trésors exposés dans les grands musées du monde, on comprend que son sens de la propriété se résume surtout à celui de s'approprier définitivement et de vendre à son profit ce qui appartenait jusqu'ici à l'espace public de la culture.

Il est cependant possible de repenser la notion de droit d'auteur en fonction du nouvel environnement virtuel, mais pour cela, il faudra cesser d'en faire un absolu et un bloc monolithique. Le principe qui devrait guider une telle révision est de sauvegarder la gratuité de la lecture et de la consultation en ligne des archives culturelles d'une société, comme le permet la bibliothèque depuis des millénaires. Son objectif est d'éviter une fracture irréversible entre la culture du Web et celle du papier, et que cette dernière ne devienne frappée d'obsolescence pour une part croissante de la population et des segments entiers de la production culturelle. Le postulat de base qui devrait guider cet aggiornamento est qu'il est nécessaire de différencier l'accès au texte en fonction du support sur lequel il est affiché et selon la possibilité qu'a le lecteur de le manipuler à sa guise, de l'annoter et de le conserver à long terme. En effet, si on ne lit pas un texte de la même façon dans un navigateur Web, un e-book ou un codex, il est normal de tenir compte de ces différences pour moduler la nature et la durée de la protection accordée à l'éditeur. 
Si l'on examine la rotation des titres sur les étalages des librairies, force est de constater que la carrière d'un livre est devenue très éphémère et que celui-ci réalise l'essentiel de ses ventes dans les quelques mois qui suivent sa publication. Il serait donc sans doute acceptable, tout en sauvegardant les dispositions actuelles du droit d'auteur dans le monde physique, de permettre à la bibliothèque nationale, de concert avec les maisons d'édition, de rendre accessible sur un site Web public, dans un format minimal et sans possibilité d'appropriation commerciale, toute œuvre publiée. Un délai d'exclusion virtuelle publique serait maintenu par rapport au monde physique, afin de permettre aux versions commerciales d'atteindre leur seuil de rentabilité. Ce délai pourrait être de cinq ans pour les ouvrages de sciences humaines et peut-être le double pour les œuvres littéraires. Le site Gallica de la BNF jouerait ainsi pleinement son rôle de bibliothèque virtuelle, où les principales productions de la culture seraient accessibles et se fondraient dans le vaste système de renvois de la Toile universelle. Les textes publiés dans ce nouvel espace ne seraient pas isolés de ceux de la filière imprimée, ce qui permettrait à ces derniers de bénéficier de la dynamique du nouveau médium, et aux divers registres de diffusion de la culture de fonctionner en complémentarité. Le lecteur pourrait ainsi découvrir des ouvrages auxquels il n'aurait jamais pensé autrement et butiner à travers leur contenu, tout comme il peut le faire dans une librairie ou une bibliothèque. S’il jugeait l'ouvrage assez intéressant pour désirer en faire la lecture dans des conditions plus propices, il aurait alors le choix entre diverses solutions : (a) se procurer un exemplaire papier, si celui-ci est disponible; (b) faire imprimer l'ouvrage dans une librairie dotée d'un service d'impression sur demande : selon une étude récente, ce système devrait bientôt être tout à fait courant et couvrir la moitié de la production imprimée en 2020 [35]; (c) télécharger l'ouvrage en format livre électronique sur sa tablette (e-book) ou, bientôt, sur son codex numérique (e-ink) [36]. Dans les trois cas, la copie serait évidemment payante selon une grille tarifaire établie par l'éditeur. Il incomberait à ce dernier de créer pour ses collections des mises en page et des formats de lecture accueillants et conviviaux, car un livre est autre chose que du texte affiché au kilomètre et, même sur écran, le travail de l'éditeur peut lui ajouter une valeur non négligeable.

Cette proposition, qui rejoint pour l'essentiel le mouvement lancé en faveur du lyber par Michel Valensi des Éditions de l'Éclat [37], ferait échapper à la situation paradoxale dans laquelle nous a plongés l'apparition du Web, et qui permet de trouver aujourd'hui sur ce nouveau médium quantité d'ouvrages publiés voilà un siècle ou plus, alors que sont maintenus dans leur prison de papier les textes les plus proches de nous et les plus aptes à éclairer la réflexion sur le monde actuel. Leur intégration à la mémoire vive de la Toile leur 
donnerait pourtant une nouvelle vie et une actualité plus aiguë, en les rendant enfin " cherchables " et accessibles par tous. Loin d'être étouffées par ces dispositions, la création et la recherche connaîtraient un élan nouveau en raison de la fluidité accrue dans la circulation des œuvres et de leur mise en résonance mutuelle : on sait en effet que la densité des échanges favorise le renouvellement des formes culturelles. Le nouvel espace virtuel serait ainsi le prolongement naturel de la bibliothèque dans le XXIe siècle, et sa fonction dans le partage du savoir n'entrerait plus en conflit avec des lois conçues pour le monde physique. En réduisant les inégalités culturelles, que celles-ci soient dues à des facteurs économiques, à l'éducation ou au fait de vivre à l'écart des grandes métropoles, cette " très grande bibliothèque " deviendrait la voie royale d'accès à cette société du savoir envisagée par des philosophes comme Pierre Lévy ou Michel Serres, tout en contribuant à consolider l'espace francophone.

\section{Suite : Contre les nouveaux féodalismes}

\section{Notes}

[1Philippe Quéau, “ Du bien commun mondial à l'âge de l'information ”, http://2100.org/conf_queau1.html

\section{[2] http:// www.xanadu.com}

[3] Vannevar Bush, "As we may think", Atlantic Monthly, 176 (J uly 1945), 101-108. Voir le texte intégral sur le site http://www.theatlantic.com/unbound/flashbks /computer/ bushf.htm.

[4] 9 décembre 2000, p. 37-38. Dans un numéro ultérieur, le même magazine fait état d'une recherche menée par Andrew Odlyzko des laboratoires AT\&T, selon laquelle les usagers préfèrent de loin payer par forfait plutôt que par micropaiements à la pièce (14 avril 2001, p. 61).

[5]http://jefferson.village.virginia.edu/elab/hfl0155.html. Lire aussi Noah Wardrip-Fruin, " Linking and filtering, reading and writing. The library, the web, Ted Nelson, and what's wrong with micropayment ", Intelligent agent, vol. 2, no 2, p. 32-39 (s.d.)

[6]J eremy Rifkin, L'âge de l'accès : la révolution de la nouvelle économie, Paris, La Découverte, 2000.

[7] La société du spectacle, Paris, Champ Libre, 1971. Voir http://www.multimania.com/laplage/d_spec/

[8] Une infrastructure de ce type est en cours de construction. Voir Charles Platt, " The Future Will Be Fast But Not Free ", Wired, mai 2001, p. 120-127. 
[9] Voir par exemple le site www.epinions.com

[10] Voir www.britannica.com

[11] Le Parlement européen a adopté le 14 février 2001une proposition de directive, dont on a dit qu'elle ne satisfait ni le lobby des auteurs ni celui des consommateurs. Voir le compte rendu dans Le Monde, 16 février, p. 18.

[12] Michael Geist, " Copyright holders may rule the day ", The Globe and Mail, 10 août 2000, p. T4.

[13] “ Digital copyright”, The Economist, April 7th, 2001, p. 69.

[14]Communications of the ACM, 1997, vol. 40, no 2. Voir http://www.gnu.org /philosophy/right-to-read.fr.html

[15] Bertrand Warusfel, La propriété intellectuelle et l'Internet, Paris, Flammarion, Coll. “Dominos", p. 91.

[16] Christophe Jakubyszyn, “ L'inéluctable modèle de la génération Napster ”, Le Monde, 22 février 2001.

[17] Collectif, Prêter (un livre) n'est pas voler (son auteur), Paris, Mille et une nuits, 2000, p. 75.

[18] Op. cit., p. 70.

[19] " The struggle between the cultural sphere and the commercial sphere to control both access to and the content of play is one of the defining elements of the coming era", The Age of Access, p. 7.

[20] Michel Onfray, "Contre l'incendie des bibliothèques ”, dans Prêter (un livre) n'est pas voler(son auteur).

[21] J ean-Luc Hennig, Apologie du plagiat, Gallimard, NRF, 1997.

[22] Bertrand Warusfel, id., p. 18.

[23] On trouvera de nombreuses références sur cette question dans Christian Vandendorpe, Du papyrus à l'hypertexte, Paris, La Découverte, 1999 et www.00h00.com

[24] Marc Fernandez, “Stephen King explique sa plantade ”, 18 décembre 2000, http:// www.transfert.net/fr/revue_web/article.cfm?idx_rub=94\&idx_art=3048

[25] Op. cit., p. 89. 
[26] Voir notamment D.R. Johnson et D. Post, http://www.cli.org /X0025 LBFIN.html.

[27] http://www.clark.net/pub/rothman/ boyle.htm

[28] Les Tusculanes, I, 2, 4.

[29] http:// www.cli.org/X0025_LBFIN.html [Notre traduction].

[30] http:// www.eff.org/

[31] http:// web.mit.edu/newsoffice/nr/ 2001/ocw.html

[32] http:// www.publiclibraryofscience.org/

[33] " EC turns from CDs to DVDs in probe of predatory pricing", The Globe and Mail, 12 juin 2001, p. B13.

[34] J ean-Louis Gassée, “ Microsoft en croisade contre Open Source ”, Libération, 4 mai 2001. http:// www.liberation.fr/chroniques/gassee.html

[35] Étude réalisée par la Electronic Document Systems Foundation, http:// www.edsf.org/ images/Overview.PDF

[36] Sur l'émergence prochaine de ces nouveaux supports de lecture, voir Christian Vandendorpe, "Livre virtuel ou codex numérique? Les nouveaux prétendants", Bulletin des Bibliothèques de France, no 6, 2000, p. 17-22. http://bbf.enssib.fr /bbf/html/2000 45 6/2000-6-p17-vandendorpe.xml.asp

[37] Voir notamment Olivier Blondeau et Florent Latrive (éds), Libres enfants du savoir numérique, Éditions de l’Éclat et Michel Valensi, “ Petit traité plié en dix sur le lyber ", http:// www.lyber-eclat.net/lyber/lybertxt.html. A noter que la réflexion proposée ici a été élaborée de façon indépendante de ces textes, dont l'existence a été découverte après la rédaction d'une première version de cet article. 